\title{
RADIO TO X-RAY ENERGY DISTRIBUTION OF BL LACERTAE
}

\section{OBJECTS}

\author{
P. GIOMMI, S. G. ANSARI and A. MICOL \\ ESIS, Information Systems Division of ESA, ESRIN, Frascati, Italy
}

\begin{abstract}
.
We have constructed radio to X-ray energy distributions for a large number of BL Lacertae objects using archival data. We find that Radio to optical spectra of RBLs and XBLs are very similar. Large differences are seen at higher frequencies where RBLs very frequently show a cutoff near the optical band while XBLs usually do not show any turnover before UV/X-ray energies. Our data is consistent with a picture where RBL and XBL are from the same parent population, the XBL simply being those (rare) objects where the break in the energy distribution is located at high energy.
\end{abstract}

\section{Summary}

We present multifrequency spectra of about 110 Radio and X-ray selected BL Lacertae objects constructed using non-simultaneous archival data. The data have been collected using the European Space Information System (ESIS, Giommi \& Ansari 1993) and are from several radio catalogues, the IRAS faint source catalogue, the Veron \& Veron (91) catalogue, the Einstein and EXOSAT databases.

The radio to optical spectrum of Radio Selected (RBL) and X-ray Selected (XBL) BL Lacs are very similar. The difference between these two classes of objects is limited to the optical/X-ray part of the spectrum. The classical radio discovered BL Lacs are characterized by an energy distribution with a sharp cutoff in the IR/optical band while in most of the X-ray discovered objects the turnover is located near the UV/X-ray band or at higher frequencies. For a given radio flux this can give rise to a difference in the X-ray flux of factor of 100 or more. The population of BL Lac objects probably includes a wide range of energy cutoffs. From the radio selected sample we see that the probability that the cutoff falls in the X-Ray band (or beyond) is small and is of the order of $10 \%$. The LogN-LogS of RBL (Stickel et al. 1991) gives $N(S>1 J y)=10^{-3} \mathrm{deg}^{-2}$. Since the typical radio flux of a XBL with X-ray flux of $5 \times 10^{-13}-10^{-12} \mathrm{erg} \mathrm{cm}^{-2} \mathrm{~s}^{-1}$ is of the order of $10 \mathrm{mJy}$, the expected density of these objects is $N(S>10 \mathrm{mJy})=$ $10^{-3} \times(1000 \mathrm{mJy} / 10 \mathrm{mJy})^{1.5}=1.0 \mathrm{deg}^{-2}$ (if the Euclidean slope extends to $10 \mathrm{mJy}$ ). Assuming that about $10 \%$ of these sources have a cutoff at sufficiently high energy to be detected in the EMSS, the expected density of BL Lacs in the EMSS is $\approx 0.1 \mathrm{deg}^{-2}$ which is about the value measured (Maccacaro et al. 1989 , Giommi et al. 1989). RBL and XBL are known to populate different regions of the $\alpha_{o x} v s \alpha_{\text {ro }}$ diagram. We find that this is probably the consequence of the different position of the energy cutoff.

T. J.-L. Courvoisier and A. Blecha: Multi-Wavelength Continuum Emission of AGN, 506-507.

(C) 1994 IAU. Printed in the Netherlands. 


\section{References}

Giommi, P., et al. 1989, in "BL Lac Objects", L. Maraschi, T. Maccacaro, and M.-H. Ulrich eds., Springer-Verlag p. 231.

Giommi, P., \& Ansari 1993, Proc of XXVII ESLAB Symposium, Noordwijk, The Netherlands.

Maccacaro, T., Gioia, I. M., Schild, R. E., Wolter, A., Morris, S. L. and Stocke, J. T. 1989, in

"BL Lac Objects", L. Maraschi, T. Maccacaro, and M.-H. Ulrich eds., Springer-Verlag p. 222.

Stickel, M., Fried, J.W., Kuehr, H., Padovani, P., and Urry, C.M., 1991, APJ, 374, 431. 